\title{
Bi-implication Operator on Intuitionistic Fuzzy Set
}

\author{
P. Murugadas \\ Assistant Professor \\ Mathematics Section, FEAT \\ Annamalai University, \\ Annamalainagar, India.
}

\author{
K.Lalitha \\ Assistant Professor \\ Mathematics Section, FEAT \\ Annamalai University, \\ Annamalainagar, India.
}

\begin{abstract}
In this paper we introduce a Biimplication operator $\leftrightarrow$ for intuitionistic fuzzy set and discuss several properties of it.
\end{abstract}

\section{Keywords:}

Intuitionistic Fuzzy Sets(IFSs), Intuitionistic Fuzzy Matrix(IFM), implication operator and bi-implication operator.

\section{INTRODUCTION}

After the introduction of fuzzy set theory by Zadah[7] in 1965, fuzzy concepts evolved in almost all fields. Hiroshi Hasimoto[2] used implication operator in fuzzy set and extended it to fuzzy matrix theory and obtained results in sub-inverse of fuzzy matrix using fuzzy relational equation. Atanassov[1] generalized fuzzy set theory to intuitionistic fuzzy set theory. S.Sriram and P.Murugadas[4,5,6] developed this implication operator to intuitionistic fuzzy set and extended it to IFM. The authors [3] have studied dual implication operators in IFS. In this paper we introduce a bi-implication operator to IFSs and study some properties of it.

Definition 1.1. [1] An Intuitionistic Fuzzy Set(IFS) $A$ in $E$ (universal set) is defined as an object of the following form $A=\left\{\left\langle x, \mu_{A}(x), \nu_{A}(x)\right\rangle / x \in E\right\}$, where the functions: $\mu_{A}(x)$ : $E \rightarrow[0,1]$ and $\nu_{A}(x): E \rightarrow[0,1]$ define the membership and non-membership of the element $x \in E$ respectively and for every $x \in E: 0 \leq \mu_{A(x)}+\nu_{A}(x) \leq 1$.

For simplicity we consider the pair $\left\langle x, x^{\prime}\right\rangle$ as membership and nonmembership function of an IFS with $x+x^{\prime} \leq 1$.

Definition 1.2. [6] Let $\left\langle a, a^{\prime}\right\rangle,\left\langle b, b^{\prime}\right\rangle \in I F S$ define $\left\langle a, a^{\prime}\right\rangle \rightarrow\left\langle b, b^{\prime}\right\rangle$

$= \begin{cases}\langle 1,0\rangle & \text { if }\left\langle a, a^{\prime}\right\rangle \leq\left\langle b, b^{\prime}\right\rangle \\ \left\langle b, b^{\prime}\right\rangle & \left.\text { if }\left\langle a, a^{\prime}\right\rangle\right\rangle\left\langle b, b^{\prime}\right\rangle\end{cases}$

and $\left\langle a, a^{\prime}\right\rangle \leftarrow\left\langle b, b^{\prime}\right\rangle=\left(\left\langle b, b^{\prime}\right\rangle \rightarrow\left\langle a, a^{\prime}\right\rangle\right)$.

\section{BI-IMPLICATION OPERATOR $\leftrightarrow$}

DEFINITION 2.1. Let $\left\langle a, a^{\prime}\right\rangle,\left\langle b, b^{\prime}\right\rangle \in I F S$ define $\left\langle a, a^{\prime}\right\rangle \leftrightarrow\left\langle b, b^{\prime}\right\rangle=\left(\left\langle a, a^{\prime}\right\rangle \leftarrow\left\langle b, b^{\prime}\right\rangle\right) \wedge\left(\left\langle a, a^{\prime}\right\rangle \rightarrow\left\langle b, b^{\prime}\right\rangle\right)$ that is

$$
\left\langle a, a^{\prime}\right\rangle \leftrightarrow\left\langle b, b^{\prime}\right\rangle=\left\{\begin{array}{lll}
\left\langle b, b^{\prime}\right\rangle & \text { if }\left\langle a, a^{\prime}\right\rangle>\left\langle b, b^{\prime}\right\rangle \\
\langle 1,0\rangle & \text { if }\left\langle a, a^{\prime}\right\rangle=\left\langle b, b^{\prime}\right\rangle \\
\left\langle a, a^{\prime}\right\rangle & \text { if }\left\langle a, a^{\prime}\right\rangle<\left\langle b, b^{\prime}\right\rangle
\end{array}\right.
$$

Easily $\left\langle a, a^{\prime}\right\rangle \leftrightarrow\left\langle b, b^{\prime}\right\rangle=\left\langle b, b^{\prime}\right\rangle \leftrightarrow\left\langle a, a^{\prime}\right\rangle$.

Proposition 2.2. Let $\left\langle a, a^{\prime}\right\rangle,\left\langle b, b^{\prime}\right\rangle,\left\langle c, c^{\prime}\right\rangle \in I F S$, then $\left(\left\langle a, a^{\prime}\right\rangle \leftrightarrow\left\langle c, c^{\prime}\right\rangle\right) \wedge\left(\left\langle b, b^{\prime}\right\rangle \leftrightarrow\left\langle c, c^{\prime}\right\rangle\right) \leq\left(\left\langle a, a^{\prime}\right\rangle \leftrightarrow\left\langle b, b^{\prime}\right\rangle\right)$

PROOF.

case 1. If $\left\langle c, c^{\prime}\right\rangle \neq\left\langle a, a^{\prime}\right\rangle,\left\langle b, b^{\prime}\right\rangle$ then $\left(\left\langle a, a^{\prime}\right\rangle \leftrightarrow\left\langle c, c^{\prime}\right\rangle\right) \wedge$ $\left(\left\langle b, b^{\prime}\right\rangle \leftrightarrow\left\langle c, c^{\prime}\right\rangle\right)=\left\langle a, a^{\prime}\right\rangle \wedge\left\langle b, b^{\prime}\right\rangle \wedge\left\langle c, c^{\prime}\right\rangle \leq\left\langle a, a^{\prime}\right\rangle \wedge\left\langle b, b^{\prime}\right\rangle \leq$ $\left\langle a, a^{\prime}\right\rangle \leftrightarrow\left\langle b, b^{\prime}\right\rangle$

case 2. If $\left\langle c, c^{\prime}\right\rangle=\left\langle a, a^{\prime}\right\rangle$ but $\left\langle c, c^{\prime}\right\rangle \neq\left\langle b, b^{\prime}\right\rangle$ then $\left(\left\langle a, a^{\prime}\right\rangle \leftrightarrow\right.$ $\left.\left\langle c, c^{\prime}\right\rangle\right) \wedge\left(\left\langle b, b^{\prime}\right\rangle \leftrightarrow\left\langle c, c^{\prime}\right\rangle\right)=\left(\left\langle b, b^{\prime}\right\rangle \leftrightarrow\left\langle c, c^{\prime}\right\rangle\right)=\left(\left\langle b, b^{\prime}\right\rangle \leftrightarrow\right.$ $\left.\left\langle a, a^{\prime}\right\rangle\right)=\left(\left\langle a, a^{\prime}\right\rangle \leftrightarrow\left\langle b, b^{\prime}\right\rangle\right)$

case 3. If $\left\langle c, c^{\prime}\right\rangle \neq\left\langle a, a^{\prime}\right\rangle$ but $\left\langle c, c^{\prime}\right\rangle=\left\langle b, b^{\prime}\right\rangle$ then $\left(\left\langle a, a^{\prime}\right\rangle \leftrightarrow\right.$ $\langle c, c\rangle) \wedge\left(\left\langle b, b^{\prime}\right\rangle \leftrightarrow\left\langle c, c^{\prime}\right\rangle\right)=\left\langle a, a^{\prime}\right\rangle \leftrightarrow\left\langle c, c^{\prime}\right\rangle=\left\langle a, a^{\prime}\right\rangle \leftrightarrow\left\langle b, b^{\prime}\right\rangle$ case 4. If $\left\langle a, a^{\prime}\right\rangle=\left\langle b, b^{\prime}\right\rangle=\left\langle c, c^{\prime}\right\rangle$ then $\left(\left\langle a, a^{\prime}\right\rangle \leftrightarrow\left\langle c, c^{\prime}\right\rangle\right) \wedge$ $\left(\left\langle b, b^{\prime}\right\rangle \leftrightarrow\left\langle c, c^{\prime}\right\rangle\right)=\left(\left\langle a, a^{\prime}\right\rangle \leftrightarrow\left\langle b, b^{\prime}\right\rangle\right) \wedge\left(\left\langle c, c^{\prime}\right\rangle \leftrightarrow\left\langle c, c^{\prime}\right\rangle\right)=$ $\left\langle a, a^{\prime}\right\rangle \leftrightarrow\left\langle b, b^{\prime}\right\rangle$

Proposition 2.3. Let $\left\langle a, a^{\prime}\right\rangle,\left\langle b, b^{\prime}\right\rangle \in I F S$, then $\left\langle a, a^{\prime}\right\rangle \wedge\left(\left\langle a, a^{\prime}\right\rangle \leftrightarrow\left\langle b, b^{\prime}\right\rangle\right) \leq\left\langle b, b^{\prime}\right\rangle$

PROOF.

$$
\begin{aligned}
& \text { PROOF. } \\
& \begin{aligned}
\left\langle a, a^{\prime}\right\rangle \wedge\left(\left\langle a, a^{\prime}\right\rangle \leftrightarrow\left\langle b, b^{\prime}\right\rangle\right) & = \begin{cases}\left\langle b, b^{\prime}\right\rangle & \text { if }\left\langle a, a^{\prime}\right\rangle>\left\langle b, b^{\prime}\right\rangle \\
\left\langle a, a^{\prime}\right\rangle & \text { if }\left\langle a, a^{\prime}\right\rangle \leq\left\langle b, b^{\prime}\right\rangle\end{cases} \\
& \leq\left\langle b, b^{\prime}\right\rangle . \quad \square
\end{aligned}
\end{aligned}
$$

LEMMA 2.4

$\left(\left\langle a, a^{\prime}\right\rangle \wedge\left\langle b, b^{\prime}\right\rangle\right) \leftarrow\left\langle c, c^{\prime}\right\rangle=\left(\left\langle a, a^{\prime}\right\rangle \leftarrow\left\langle c, c^{\prime}\right\rangle\right) \wedge\left(\left\langle b, b^{\prime}\right\rangle \leftarrow\left\langle c, c^{\prime}\right\rangle\right)$

Proof. case 1

Assume $\left\langle a, a^{\prime}\right\rangle<\left\langle b, b^{\prime}\right\rangle$

sub case 1 .

If $\left\langle b, b^{\prime}\right\rangle<\left\langle c, c^{\prime}\right\rangle$, then $\left\langle a, a^{\prime}\right\rangle<\left\langle c, c^{\prime}\right\rangle$, so

$\left\langle a, a^{\prime}\right\rangle \leftarrow\left\langle c, c^{\prime}\right\rangle=\left\langle a, a^{\prime}\right\rangle \wedge\left\langle b, b^{\prime}\right\rangle$

$\left\langle a, a^{\prime}\right\rangle=\left\langle a, a^{\prime}\right\rangle$.

sub case 2 . If $\left\langle a, a^{\prime}\right\rangle\left\langle\left\langle c, c^{\prime}\right\rangle\right.$, then $\left\langle b, b^{\prime}\right\rangle>\left\langle c, c^{\prime}\right\rangle$ or $\left\langle b, b^{\prime}\right\rangle<$ $\left\langle c, c^{\prime}\right\rangle$ consider $\left\langle b, b^{\prime}\right\rangle>\left\langle c, c^{\prime}\right\rangle$, then 
$\left\langle a, a^{\prime}\right\rangle \leftarrow\left\langle c, c^{\prime}\right\rangle=\left\langle a, a^{\prime}\right\rangle \wedge\langle 1,0\rangle,\left\langle a, a^{\prime}\right\rangle=\left\langle a, a^{\prime}\right\rangle$. Similarly we can prove when $\left\langle b, b^{\prime}\right\rangle\left\langle\left\langle c, c^{\prime}\right\rangle\right.$

sub case 3. If $\left\langle c, c^{\prime}\right\rangle \neq\left\langle a, a^{\prime}\right\rangle$ and $\left\langle c, c^{\prime}\right\rangle=\left\langle b, b^{\prime}\right\rangle$

$\left\langle a, a^{\prime}\right\rangle \leftarrow\left\langle c, c^{\prime}\right\rangle=\left(\left\langle a, a^{\prime}\right\rangle \leftarrow\left\langle b, b^{\prime}\right\rangle\right) \wedge\left(\left\langle c, c^{\prime}\right\rangle \leftarrow\left\langle c, c^{\prime}\right\rangle\right)$

$\left\langle a, a^{\prime}\right\rangle \leftarrow\left\langle b, b^{\prime}\right\rangle=\left(\left\langle a, a^{\prime}\right\rangle \leftarrow\left\langle b, b^{\prime}\right\rangle\right) \wedge\langle 1,0\rangle$

$\left\langle a, a^{\prime}\right\rangle=\left\langle a, a^{\prime}\right\rangle \wedge\langle 1,0\rangle$

$\left\langle a, a^{\prime}\right\rangle=\left\langle a, a^{\prime}\right\rangle$.

sub case 4. If $\left\langle c, c^{\prime}\right\rangle=\left\langle a, a^{\prime}\right\rangle$ and $\left\langle c, c^{\prime}\right\rangle \neq\left\langle b, b^{\prime}\right\rangle$

$\left\langle a, a^{\prime}\right\rangle \leftarrow\left\langle c, c^{\prime}\right\rangle=\left(\left\langle a, a^{\prime}\right\rangle \leftarrow\left\langle a, a^{\prime}\right\rangle\right) \wedge\left(\left\langle b, b^{\prime}\right\rangle \leftarrow\left\langle a, a^{\prime}\right\rangle\right)$

$\left\langle a, a^{\prime}\right\rangle \leftarrow\left\langle a, a^{\prime}\right\rangle=\langle 1,0\rangle \wedge\langle 1,0\rangle$

$\langle 1,0\rangle=\langle 1,0\rangle$. Hence $(2.1)$ holds

case 2 . Assume $\left.\left\langle a, a^{\prime}\right\rangle\right\rangle\left\langle b, b^{\prime}\right\rangle$

sub case 1. If $\left\langle b, b^{\prime}\right\rangle>\left\langle c, c^{\prime}\right\rangle$ then $\left\langle a, a^{\prime}\right\rangle>\left\langle c, c^{\prime}\right\rangle,\left\langle b, b^{\prime}\right\rangle \leftarrow$ $\left\langle c, c^{\prime}\right\rangle=\langle 1,0\rangle \wedge\langle 1,0\rangle,\langle 1,0\rangle=\langle 1,0\rangle$

sub case 2. If $\left.\left\langle a, a^{\prime}\right\rangle\right\rangle\left\langle c, c^{\prime}\right\rangle$ and $\left\langle b, b^{\prime}\right\rangle<\left\langle c, c^{\prime}\right\rangle,\left\langle b, b^{\prime}\right\rangle \leftarrow$ $\left\langle c, c^{\prime}\right\rangle=\langle 1,0\rangle \wedge\left\langle b, b^{\prime}\right\rangle,\left\langle b, b^{\prime}\right\rangle=\left\langle b, b^{\prime}\right\rangle$

sub case 3. If $\left\langle a, a^{\prime}\right\rangle<\left\langle c, c^{\prime}\right\rangle$ then $\left\langle b, b^{\prime}\right\rangle<\left\langle c, c^{\prime}\right\rangle,\left\langle b, b^{\prime}\right\rangle \leftarrow$ $\left\langle c, c^{\prime}\right\rangle=\left\langle a, a^{\prime}\right\rangle \wedge\left\langle b, b^{\prime}\right\rangle,\left\langle b, b^{\prime}\right\rangle=\left\langle b, b^{\prime}\right\rangle$

sub case 4. if $\left\langle c, c^{\prime}\right\rangle \neq\left\langle a, a^{\prime}\right\rangle$ and $\left\langle c, c^{\prime}\right\rangle=\left\langle b, b^{\prime}\right\rangle,\left\langle b, b^{\prime}\right\rangle \leftarrow$ $\left\langle c, c^{\prime}\right\rangle=\left(\left\langle a, a^{\prime}\right\rangle \leftarrow\left\langle b, b^{\prime}\right\rangle\right) \wedge\left(\left\langle b, b^{\prime}\right\rangle \leftarrow\left\langle b, b^{\prime}\right\rangle\right),\left\langle b, b^{\prime}\right\rangle \leftarrow\left\langle b, b^{\prime}\right\rangle=$ $\langle 1,0\rangle \wedge\langle 1,0\rangle\langle 1,0\rangle=\langle 1,0\rangle$

sub case 5. if $\left\langle c, c^{\prime}\right\rangle=\left\langle a, a^{\prime}\right\rangle$ and $\left\langle c, c^{\prime}\right\rangle \neq\left\langle b, b^{\prime}\right\rangle,\left\langle b, b^{\prime}\right\rangle \leftarrow$ $\left\langle c, c^{\prime}\right\rangle=\left(\left\langle a, a^{\prime}\right\rangle \leftarrow\left\langle a, a^{\prime}\right\rangle\right) \wedge\left(\left\langle b, b^{\prime}\right\rangle \leftarrow\left\langle a, a^{\prime}\right\rangle\right),\left\langle b, b^{\prime}\right\rangle \leftarrow$ $\left\langle a, a^{\prime}\right\rangle=\langle 1,0\rangle \wedge\left\langle b, b^{\prime}\right\rangle,\left\langle b, b^{\prime}\right\rangle=\left\langle b, b^{\prime}\right\rangle$.

Hence (2.1) holds.

REMARK 2.5. Lemma(2.1)Can be generalized as $\left(\bigwedge_{i}\left\langle a_{i}, a_{i}^{\prime}\right\rangle\right) \leftarrow\left\langle c, c^{\prime}\right\rangle=\bigwedge_{i}\left(\left\langle a_{i}, a_{i}^{\prime}\right\rangle \leftarrow\left\langle c, c^{\prime}\right\rangle\right)$

LEMMA 2.6. 1. $\left(\left\langle a, a^{\prime}\right\rangle \wedge\left\langle b, b^{\prime}\right\rangle\right) \rightarrow\left\langle c, c^{\prime}\right\rangle=\left(\left\langle a, a^{\prime}\right\rangle \rightarrow\right.$ $\left.\left\langle c, c^{\prime}\right\rangle\right) \vee\left(\left\langle b, b^{\prime}\right\rangle \rightarrow\left\langle c, c^{\prime}\right\rangle\right)$

Proof. case 1. assume $\left\langle a, a^{\prime}\right\rangle\left\langle\left\langle b, b^{\prime}\right\rangle\right.$

sub case 1. If $\left\langle b, b^{\prime}\right\rangle\left\langle\left\langle c, c^{\prime}\right\rangle\right.$, then $\left\langle a, a^{\prime}\right\rangle\left\langle\left\langle c, c^{\prime}\right\rangle,\left\langle a, a^{\prime}\right\rangle \rightarrow\right.$ $\left\langle c, c^{\prime}\right\rangle=\langle 1,0\rangle \vee\langle 1,0\rangle,\langle 1,0\rangle=\langle 1,0\rangle$.

sub case 2. If $\left\langle a, a^{\prime}\right\rangle>\left\langle c, c^{\prime}\right\rangle$ then $\left\langle b, b^{\prime}\right\rangle>\left\langle c, c^{\prime}\right\rangle,\left\langle a, a^{\prime}\right\rangle \rightarrow$ $\left\langle c, c^{\prime}\right\rangle=\left\langle c, c^{\prime}\right\rangle \vee\left\langle c, c^{\prime}\right\rangle,\left\langle c, c^{\prime}\right\rangle=\left\langle c, c^{\prime}\right\rangle$

sub case 3. If $\left\langle a, a^{\prime}\right\rangle\left\langle\left\langle c, c^{\prime}\right\rangle\right.$ and $\left\langle b, b^{\prime}\right\rangle>\left\langle c, c^{\prime}\right\rangle,\left\langle a, a^{\prime}\right\rangle \rightarrow$ $\left\langle c, c^{\prime}\right\rangle=\left(\langle 1,0\rangle \vee\left\langle c, c^{\prime}\right\rangle\right),\langle 1,0\rangle=\langle 1,0\rangle$

sub case 4 . If $\left\langle c, c^{\prime}\right\rangle \neq\left\langle a, a^{\prime}\right\rangle$ and $\left\langle c, c^{\prime}\right\rangle=\left\langle b, b^{\prime}\right\rangle,\left\langle a, a^{\prime}\right\rangle \rightarrow$ $\left\langle c, c^{\prime}\right\rangle=\left(\left\langle a, a^{\prime}\right\rangle \rightarrow\left\langle b, b^{\prime}\right\rangle\right) \vee\left(\left\langle b, b^{\prime}\right\rangle \rightarrow\left\langle b, b^{\prime}\right\rangle\right),\left\langle a, a^{\prime}\right\rangle \rightarrow$ $\left\langle b, b^{\prime}\right\rangle=\langle 1,0\rangle \vee\langle 1,0\rangle,\langle 1,0\rangle=\langle 1,0\rangle$

sub case 5. If $\left\langle c, c^{\prime}\right\rangle=\left\langle a, a^{\prime}\right\rangle$ and $\left\langle c, c^{\prime}\right\rangle \neq\left\langle b, b^{\prime}\right\rangle,\left\langle a, a^{\prime}\right\rangle \rightarrow$ $\left\langle c, c^{\prime}\right\rangle=\left(\left\langle a, a^{\prime}\right\rangle \rightarrow\left\langle a, a^{\prime}\right\rangle\right) \vee\left(\left\langle b, b^{\prime}\right\rangle \rightarrow\left\langle a, a^{\prime}\right\rangle\right),\left\langle a, a^{\prime}\right\rangle \rightarrow$ $\left\langle a, a^{\prime}\right\rangle=\langle 1,0\rangle \vee\left\langle a, a^{\prime}\right\rangle,\langle 1,0\rangle=\langle 1,0\rangle$

case 2 . Assume $\left.\left\langle a, a^{\prime}\right\rangle\right\rangle\left\langle b, b^{\prime}\right\rangle$

sub case 1. If $\left\langle a, a^{\prime}\right\rangle<\left\langle c, c^{\prime}\right\rangle$ then $\left\langle b, b^{\prime}\right\rangle<\left\langle c, c^{\prime}\right\rangle,\left\langle b, b^{\prime}\right\rangle \rightarrow$ $\left\langle c, c^{\prime}\right\rangle=\langle 1,0\rangle \vee\langle 1,0\rangle,\langle 1,0\rangle=\langle 1,0\rangle$

sub case 2. If $\left.\left\langle a, a^{\prime}\right\rangle\right\rangle\left\langle c, c^{\prime}\right\rangle$ and $\left\langle b, b^{\prime}\right\rangle\left\langle\left\langle c, c^{\prime}\right\rangle,\left\langle b, b^{\prime}\right\rangle \rightarrow\right.$ $\left\langle c, c^{\prime}\right\rangle=\left\langle c, c^{\prime}\right\rangle \vee\langle 1,0\rangle,\langle 1,0\rangle=\langle 1,0\rangle$

sub case 3. If $\left\langle c, c^{\prime}\right\rangle \neq\left\langle a, a^{\prime}\right\rangle$ and $\left\langle c, c^{\prime}\right\rangle=\left\langle b, b^{\prime}\right\rangle,\left\langle b, b^{\prime}\right\rangle \rightarrow$ $\left\langle c, c^{\prime}\right\rangle=\left(\left\langle a, a^{\prime}\right\rangle \rightarrow\left\langle b, b^{\prime}\right\rangle\right) \vee\left(\left\langle b, b^{\prime}\right\rangle \rightarrow\left\langle b, b^{\prime}\right\rangle\right),\left\langle b, b^{\prime}\right\rangle \rightarrow\left\langle b, b^{\prime}\right\rangle=$ $\langle 1,0\rangle \vee\langle 1,0\rangle,\langle 1,0\rangle=\langle 1,0\rangle$

sub case 4. If $\left\langle c, c^{\prime}\right\rangle=\left\langle a, a^{\prime}\right\rangle$ and $\left\langle c, c^{\prime}\right\rangle \neq\left\langle b, b^{\prime}\right\rangle,\left\langle b, b^{\prime}\right\rangle \rightarrow$ $\left\langle c, c^{\prime}\right\rangle=\left(\left\langle a, a^{\prime}\right\rangle \rightarrow\left\langle a, a^{\prime}\right\rangle\right) \vee\left(\left\langle b, b^{\prime}\right\rangle \rightarrow\left\langle a, a^{\prime}\right\rangle\right),\left\langle b, b^{\prime}\right\rangle \rightarrow$ $\left\langle a, a^{\prime}\right\rangle=\langle 1,0\rangle \vee\langle 1,0\rangle,\langle 1,0\rangle=\langle 1,0\rangle$.

sub case 5. If $\left\langle a, a^{\prime}\right\rangle>\left\langle c, c^{\prime}\right\rangle$ and $\left\langle b, b^{\prime}\right\rangle>\left\langle c, c^{\prime}\right\rangle$

then $\left\langle b, b^{\prime}\right\rangle \rightarrow\left\langle c, c^{\prime}\right\rangle$

$=\left(\left\langle a, a^{\prime}\right\rangle \rightarrow\left\langle c, c^{\prime}\right\rangle\right) \vee\left(\left\langle b, b^{\prime}\right\rangle \rightarrow\left\langle c, c^{\prime}\right\rangle\right)$

$\Rightarrow\left\langle c, c^{\prime}\right\rangle$

$=\left\langle c, c^{\prime}\right\rangle \vee\left\langle c, c^{\prime}\right\rangle$

$=\left\langle c, c^{\prime}\right\rangle$.

Thus Lemma (2.2) holds.
REMARK 2.7. Can be generalized as $\left(\bigwedge_{i=1}^{n}\left\langle a_{i}, a_{i}^{\prime}\right\rangle\right) \rightarrow$ $\left\langle c, c^{\prime}\right\rangle=\bigvee_{i=1}^{n}\left(\left\langle a_{i}, a_{i}^{\prime}\right\rangle \rightarrow\left\langle c, c^{\prime}\right\rangle\right)$

LEMMA 2.8

$\left(\left\langle a, a^{\prime}\right\rangle \vee\left\langle b, b^{\prime}\right\rangle\right) \rightarrow\left\langle c, c^{\prime}\right\rangle=\left(\left\langle a, a^{\prime}\right\rangle \rightarrow\left\langle c, c^{\prime}\right\rangle\right) \wedge\left(\left\langle b, b^{\prime}\right\rangle \rightarrow\right.$ $\left.\left\langle c, c^{\prime}\right\rangle\right)$

Proof. case 1. Assume $\left\langle a, a^{\prime}\right\rangle<\left\langle b, b^{\prime}\right\rangle$

sub case 1. If $\left\langle b, b^{\prime}\right\rangle\left\langle\left\langle c, c^{\prime}\right\rangle\right.$, then $\left\langle a, a^{\prime}\right\rangle\left\langle\left\langle c, c^{\prime}\right\rangle\right.$

$\left\langle b, b^{\prime}\right\rangle \rightarrow\left\langle c, c^{\prime}\right\rangle=\langle 1,0\rangle \wedge\langle 1,0\rangle$

$\langle 1,0\rangle=\langle 1,0\rangle$

sub case 2 . If $\left\langle a, a^{\prime}\right\rangle>\left\langle c, c^{\prime}\right\rangle$ and $\left\langle b, b^{\prime}\right\rangle>\left\langle c, c^{\prime}\right\rangle$

$\left\langle b, b^{\prime}\right\rangle \rightarrow\left\langle c, c^{\prime}\right\rangle=\left\langle c, c^{\prime}\right\rangle \wedge\left\langle c, c^{\prime}\right\rangle$

$\left\langle c, c^{\prime}\right\rangle=\left\langle c, c^{\prime}\right\rangle$

sub case 3. If $\left\langle a, a^{\prime}\right\rangle\left\langle\left\langle c, c^{\prime}\right\rangle\right.$ and $\left.\left\langle b, b^{\prime}\right\rangle\right\rangle\left\langle c, c^{\prime}\right\rangle$

$\left\langle b, b^{\prime}\right\rangle \rightarrow\left\langle c, c^{\prime}\right\rangle=\left(\langle 1,0\rangle \wedge\left\langle c, c^{\prime}\right\rangle\right)$

$\left\langle c, c^{\prime}\right\rangle=\left\langle c, c^{\prime}\right\rangle$

sub case 4 . If $\left\langle c, c^{\prime}\right\rangle \neq\left\langle a, a^{\prime}\right\rangle$ and $\left\langle c, c^{\prime}\right\rangle=\left\langle b, b^{\prime}\right\rangle$

$\left\langle b, b^{\prime}\right\rangle \rightarrow\left\langle c, c^{\prime}\right\rangle=\left(\left\langle a, a^{\prime}\right\rangle \rightarrow\left\langle b, b^{\prime}\right\rangle\right) \wedge\left(\left\langle b, b^{\prime}\right\rangle \rightarrow\left\langle b, b^{\prime}\right\rangle\right)$

$\left\langle b, b^{\prime}\right\rangle \rightarrow\left\langle b, b^{\prime}\right\rangle=\langle 1,0\rangle \wedge\langle 1,0\rangle$

$\langle 1,0\rangle=\langle 1,0\rangle$

sub case 5 . If $\left\langle c, c^{\prime}\right\rangle=\left\langle a, a^{\prime}\right\rangle$ and $\left\langle c, c^{\prime}\right\rangle \neq\left\langle b, b^{\prime}\right\rangle$

$\left\langle b, b^{\prime}\right\rangle \rightarrow\left\langle c, c^{\prime}\right\rangle=\left(\left\langle a, a^{\prime}\right\rangle \rightarrow\left\langle a, a^{\prime}\right\rangle\right) \wedge\left(\left\langle b, b^{\prime}\right\rangle \rightarrow\left\langle a, a^{\prime}\right\rangle\right)$

$\left\langle b, b^{\prime}\right\rangle \rightarrow\left\langle a, a^{\prime}\right\rangle=\langle 1,0\rangle \wedge\left\langle a, a^{\prime}\right\rangle$

$\left\langle a, a^{\prime}\right\rangle=\left\langle a, a^{\prime}\right\rangle$

case 2.

Assume $\left\langle a, a^{\prime}\right\rangle>\left\langle b, b^{\prime}\right\rangle$

sub case 1 . If $\left\langle b, b^{\prime}\right\rangle\left\langle\left\langle c, c^{\prime}\right\rangle\right.$ and $\left\langle a, a^{\prime}\right\rangle\left\langle\left\langle c, c^{\prime}\right\rangle\right.$

$\left\langle a, a^{\prime}\right\rangle \rightarrow\left\langle c, c^{\prime}\right\rangle=\langle 1,0\rangle \wedge\langle 1,0\rangle$

$\langle 1,0\rangle=\langle 1,0\rangle$

sub case 2. If $\left.\left\langle a, a^{\prime}\right\rangle\right\rangle\left\langle c, c^{\prime}\right\rangle$ and $\left\langle b, b^{\prime}\right\rangle\left\langle\left\langle c, c^{\prime}\right\rangle\right.$

$\left\langle a, a^{\prime}\right\rangle \rightarrow\left\langle c, c^{\prime}\right\rangle=\left\langle c, c^{\prime}\right\rangle \wedge\langle 1,0\rangle$

$\left\langle c, c^{\prime}\right\rangle=\left\langle c, c^{\prime}\right\rangle$

sub case 3. If $\left\langle c, c^{\prime}\right\rangle \neq\left\langle a, a^{\prime}\right\rangle$ and $\left\langle c, c^{\prime}\right\rangle=\left\langle b, b^{\prime}\right\rangle$

$\left\langle a, a^{\prime}\right\rangle \rightarrow\left\langle c, c^{\prime}\right\rangle=\left(\left\langle a, a^{\prime}\right\rangle \rightarrow\left\langle b, b^{\prime}\right\rangle\right) \wedge\left(\left\langle b, b^{\prime}\right\rangle \rightarrow\left\langle b, b^{\prime}\right\rangle\right)$

$\left\langle a, a^{\prime}\right\rangle \rightarrow\left\langle b, b^{\prime}\right\rangle=\left\langle b, b^{\prime}\right\rangle \wedge\langle 1,0\rangle$

$\left\langle b, b^{\prime}\right\rangle=\left\langle b, b^{\prime}\right\rangle$

sub case 4 . If $\left\langle c, c^{\prime}\right\rangle=\left\langle a, a^{\prime}\right\rangle$ and $\left\langle c, c^{\prime}\right\rangle \neq\left\langle b, b^{\prime}\right\rangle$

$\left\langle a, a^{\prime}\right\rangle \rightarrow\left\langle c, c^{\prime}\right\rangle=\left(\left\langle a, a^{\prime}\right\rangle \rightarrow\left\langle a, a^{\prime}\right\rangle\right) \wedge\left(\left\langle b, b^{\prime}\right\rangle \rightarrow\left\langle b, b^{\prime}\right\rangle\right)$

$\left\langle a, a^{\prime}\right\rangle \rightarrow\left\langle a, a^{\prime}\right\rangle=\langle 1,0\rangle \wedge\langle 1,0\rangle$

$\langle 1,0\rangle=\langle 1,0\rangle$.

Thus Lemma (2.3) holds.

REMARK 2.9. Can be generalized as $\left(\bigvee_{i=1}^{n}\left\langle a_{i}, a_{i}^{\prime}\right\rangle\right) \rightarrow$ $\left\langle c, c^{\prime}\right\rangle=\bigwedge_{i=1}^{n}\left(\left\langle a_{i}, a_{i}^{\prime}\right\rangle \rightarrow\left\langle c, c^{\prime}\right\rangle\right)$

LEMMA 2.10 .

$\left(\left\langle a, a^{\prime}\right\rangle \vee\left\langle b, b^{\prime}\right\rangle\right) \leftarrow\left\langle c, c^{\prime}\right\rangle=\left(\left\langle a, a^{\prime}\right\rangle \leftarrow\left\langle c, c^{\prime}\right\rangle\right) \vee\left(\left\langle b, b^{\prime}\right\rangle \leftarrow\right.$ $\left.\left\langle c, c^{\prime}\right\rangle\right)$

Proof. case 1. Assume $\left\langle a, a^{\prime}\right\rangle\left\langle\left\langle b, b^{\prime}\right\rangle\right.$ sub case 1. If $\left\langle b, b^{\prime}\right\rangle\left\langle\left\langle c, c^{\prime}\right\rangle\right.$ then $\left\langle a, a^{\prime}\right\rangle\left\langle\left\langle c, c^{\prime}\right\rangle\right.$

$\left\langle b, b^{\prime}\right\rangle \leftarrow\left\langle c, c^{\prime}\right\rangle=\left\langle a, a^{\prime}\right\rangle \vee\left\langle b, b^{\prime}\right\rangle$

$\left\langle b, b^{\prime}\right\rangle=\left\langle b, b^{\prime}\right\rangle$

sub case 2 . If $\left\langle a, a^{\prime}\right\rangle>\left\langle c, c^{\prime}\right\rangle$ then $\left\langle b, b^{\prime}\right\rangle>\left\langle c, c^{\prime}\right\rangle$

$\left\langle b, b^{\prime}\right\rangle \leftarrow\left\langle c, c^{\prime}\right\rangle=\langle 1,0\rangle \vee\langle 1,0\rangle$

$\langle 1,0\rangle=\langle 1,0\rangle$

sub case 3. If $\left\langle a, a^{\prime}\right\rangle\left\langle\left\langle c, c^{\prime}\right\rangle\right.$ and $\left.\left\langle b, b^{\prime}\right\rangle\right\rangle\left\langle c, c^{\prime}\right\rangle$

$\left\langle b, b^{\prime}\right\rangle \leftarrow\left\langle c, c^{\prime}\right\rangle=\left(\left\langle a, a^{\prime}\right\rangle \vee\langle 1,0\rangle\right)$

$\langle 1,0\rangle=\langle 1,0\rangle$ 
sub case 4. If $\left\langle c, c^{\prime}\right\rangle \neq\left\langle a, a^{\prime}\right\rangle$ and $\left\langle c, c^{\prime}\right\rangle=\left\langle b, b^{\prime}\right\rangle$

$\left\langle b, b^{\prime}\right\rangle \leftarrow\left\langle c, c^{\prime}\right\rangle=\left(\left\langle a, a^{\prime}\right\rangle \leftarrow\left\langle b, b^{\prime}\right\rangle\right) \vee\left(\left\langle b, b^{\prime}\right\rangle \leftarrow\left\langle b, b^{\prime}\right\rangle\right)$

$\left\langle b, b^{\prime}\right\rangle \leftarrow\left\langle b, b^{\prime}\right\rangle=\left\langle a, a^{\prime}\right\rangle \vee\langle 1,0\rangle$

$\langle 1,0\rangle=\langle 1,0\rangle$

sub case 5. If $\left\langle c, c^{\prime}\right\rangle=\left\langle a, a^{\prime}\right\rangle$ and $\left\langle c, c^{\prime}\right\rangle \neq\left\langle b, b^{\prime}\right\rangle$

$\left\langle b, b^{\prime}\right\rangle \leftarrow\left\langle c, c^{\prime}\right\rangle=\left(\left\langle c, c^{\prime}\right\rangle \leftarrow\left\langle c, c^{\prime}\right\rangle\right) \vee\left(\left\langle b, b^{\prime}\right\rangle \leftarrow\left\langle a, a^{\prime}\right\rangle\right)$

$\left\langle b, b^{\prime}\right\rangle \leftarrow\left\langle a, a^{\prime}\right\rangle=\langle 1,0\rangle \vee\langle 1,0\rangle$

$\langle 1,0\rangle=\langle 1,0\rangle$

case 2 .

Assume $\left\langle a, a^{\prime}\right\rangle>\left\langle b, b^{\prime}\right\rangle$

sub case 1. If $\left\langle a, a^{\prime}\right\rangle\left\langle\left\langle c, c^{\prime}\right\rangle\right.$, then $\left\langle b, b^{\prime}\right\rangle<\left\langle c, c^{\prime}\right\rangle$

$\left\langle a, a^{\prime}\right\rangle \leftarrow\left\langle c, c^{\prime}\right\rangle=\left\langle a, a^{\prime}\right\rangle \vee\left\langle b, b^{\prime}\right\rangle$

$\left\langle a, a^{\prime}\right\rangle=\left\langle a, a^{\prime}\right\rangle$

sub case 2. If $\left.\left\langle a, a^{\prime}\right\rangle\right\rangle\left\langle c, c^{\prime}\right\rangle$ and $\left\langle b, b^{\prime}\right\rangle\left\langle\left\langle c, c^{\prime}\right\rangle\right.$

$\left\langle a, a^{\prime}\right\rangle \leftarrow\left\langle c, c^{\prime}\right\rangle=\langle 1,0\rangle \vee\left\langle b, b^{\prime}\right\rangle$

$\langle 1,0\rangle=\langle 1,0\rangle$

sub case 3 . If $\left\langle c, c^{\prime}\right\rangle \neq\left\langle a, a^{\prime}\right\rangle$ and $\left\langle c, c^{\prime}\right\rangle=\left\langle b, b^{\prime}\right\rangle$

$\left\langle a, a^{\prime} \leftarrow\right\rangle\left\langle c, c^{\prime}\right\rangle=\left(\left\langle a, a^{\prime}\right\rangle \leftarrow\left\langle b, b^{\prime}\right\rangle\right) \vee\left(\left\langle b, b^{\prime}\right\rangle \leftarrow\left\langle b, b^{\prime}\right\rangle\right)$

$\left\langle a, a^{\prime}\right\rangle \leftarrow\left\langle b, b^{\prime}\right\rangle=\langle 1,0\rangle \vee\langle 1,0\rangle$

$\langle 1,0\rangle=\langle 1,0\rangle$

sub case 4 . If $\left\langle c, c^{\prime}\right\rangle=\left\langle a, a^{\prime}\right\rangle$ and $\left\langle c, c^{\prime}\right\rangle \neq\left\langle b, b^{\prime}\right\rangle$

$\left\langle a, a^{\prime}\right\rangle \leftarrow\left\langle c, c^{\prime}\right\rangle=\left(\left\langle a, a^{\prime}\right\rangle \leftarrow\left\langle a, a^{\prime}\right\rangle\right) \vee\left(\left\langle b, b^{\prime}\right\rangle \leftarrow\left\langle a, a^{\prime}\right\rangle\right)$

$\left\langle a, a^{\prime}\right\rangle \leftarrow\left\langle a, a^{\prime}\right\rangle=\langle 1,0\rangle \vee\left\langle b, b^{\prime}\right\rangle$

$\langle 1,0\rangle=\langle 1,0\rangle$. Similarly we can prove when $\left.\left\langle a, a^{\prime}\right\rangle\right\rangle\left\langle c, c^{\prime}\right\rangle$ and $\left\langle b, b^{\prime}\right\rangle>\left\langle c, c^{\prime}\right\rangle$.

Thus Lemma (2.4) holds.

REMARK 2.11. Can be generalized as $\left(\bigvee_{i=1}^{n}\left\langle a_{i}, a_{i}^{\prime}\right\rangle\right) \leftarrow$ $\left\langle c, c^{\prime}\right\rangle=\bigvee_{i=1}^{n}\left(\left\langle a_{i}, a_{i}^{\prime}\right\rangle \leftarrow\left\langle c, c^{\prime}\right\rangle\right)$

Proposition 2.12. Let $\left\langle a, a^{\prime}\right\rangle,\left\langle b, b^{\prime}\right\rangle,\left\langle c, c^{\prime}\right\rangle \in$ IFS then (i). $\left(\left\langle a, a^{\prime}\right\rangle \wedge\left\langle b, b^{\prime}\right\rangle\right) \leftrightarrow\left\langle c, c^{\prime}\right\rangle=\left[\left(\left\langle b, b^{\prime}\right\rangle \leftarrow\left\langle c, c^{\prime}\right\rangle\right) \wedge\left(\left\langle a, a^{\prime}\right\rangle \leftrightarrow\right.\right.$ $\left.\left.\left\langle c, c^{\prime}\right\rangle\right)\right] \vee\left[\left(\left\langle a, a^{\prime}\right\rangle \leftarrow\left\langle c, c^{\prime}\right\rangle\right) \wedge\left(\left\langle b, b^{\prime}\right\rangle \leftrightarrow\left\langle c, c^{\prime}\right\rangle\right)\right]$

(ii). $.\left(\left\langle a, a^{\prime}\right\rangle \vee\left\langle b, b^{\prime}\right\rangle\right) \leftrightarrow\left\langle c, c^{\prime}\right\rangle=\left[\left(\left\langle a, a^{\prime}\right\rangle \leftrightarrow\left\langle c, c^{\prime}\right\rangle\right) \wedge\left(\left\langle b, b^{\prime}\right\rangle \rightarrow\right.\right.$ $\left.\left.\left\langle c, c^{\prime}\right\rangle\right)\right] \vee\left[\left(\left\langle a, a^{\prime}\right\rangle \rightarrow\left\langle c, c^{\prime}\right\rangle\right) \wedge\left(\left\langle b, b^{\prime}\right\rangle \leftrightarrow\left\langle c, c^{\prime}\right\rangle\right)\right]$

Proof. By using Lemma 2.1 and Lemma 2.2

(i). $\left(\left\langle a, a^{\prime}\right\rangle \wedge\left\langle b, b^{\prime}\right\rangle\right) \leftrightarrow\left\langle c, c^{\prime}\right\rangle=\left[\left(\left\langle a, a^{\prime}\right\rangle \wedge\left\langle b, b^{\prime}\right\rangle\right) \leftarrow\left\langle c, c^{\prime}\right\rangle\right] \wedge$ $\left[\left(\left\langle a, a^{\prime}\right\rangle \wedge\left\langle b, b^{\prime}\right\rangle\right) \rightarrow\left\langle c, c^{\prime}\right\rangle\right]$

$\left.=\left[\left\langle a, a^{\prime}\right\rangle \leftarrow\left\langle c, c^{\prime}\right\rangle\right) \wedge\left(\left\langle b, b^{\prime}\right\rangle \leftarrow\left\langle c, c^{\prime}\right\rangle\right)\right] \wedge\left[\left(\left\langle a, a^{\prime}\right\rangle \rightarrow\left\langle c, c^{\prime}\right\rangle\right) \vee\right.$

$\left.\left(\left\langle b, b^{\prime}\right\rangle \rightarrow\left\langle c, c^{\prime}\right\rangle\right)\right]$

$=\left(\left\langle a, a^{\prime}\right\rangle \leftarrow\left\langle c, c^{\prime}\right\rangle\right) \wedge\left[\left(\left\langle b, b^{\prime}\right\rangle \leftarrow\left\langle c, c^{\prime}\right\rangle\right) \wedge\left(\left\langle a, a^{\prime}\right\rangle \rightarrow\left\langle c, c^{\prime}\right\rangle\right) \vee\right.$

$\left.\left(\left\langle b, b^{\prime}\right\rangle \leftarrow\left\langle c, c^{\prime}\right\rangle\right) \wedge\left(\left\langle b, b^{\prime}\right\rangle \rightarrow\left\langle c, c^{\prime}\right\rangle\right)\right]$

$=\left(\left\langle a, a^{\prime}\right\rangle \leftarrow\left\langle c, c^{\prime}\right\rangle\right) \wedge\left[\left[\left(\left\langle b, b^{\prime}\right\rangle \leftarrow\left\langle c, c^{\prime}\right\rangle\right) \wedge\left(\left\langle a, a^{\prime}\right\rangle \rightarrow\left\langle c, c^{\prime}\right\rangle\right)\right] \vee\right.$ $\left(\left\langle b, b^{\prime}\right\rangle \leftrightarrow\left\langle c, c^{\prime}\right\rangle\right)$

$=\left[\left(\left\langle a, a^{\prime}\right\rangle \leftarrow\left\langle c, c^{\prime}\right\rangle\right) \wedge\left(\left\langle b, b^{\prime}\right\rangle \leftarrow\left\langle c, c^{\prime}\right\rangle\right) \wedge\left(\left\langle a, a^{\prime}\right\rangle \rightarrow\left\langle c, c^{\prime}\right\rangle\right)\right] \vee$ $\left[\left(\left\langle a, a^{\prime}\right\rangle \leftarrow\left\langle c, c^{\prime}\right\rangle\right) \wedge\left(\left\langle b, b^{\prime}\right\rangle \leftrightarrow\left\langle c, c^{\prime}\right\rangle\right)\right]$

$=\left[\left(\left\langle a, a^{\prime}\right\rangle \leftrightarrow\left\langle c, c^{\prime}\right\rangle\right) \wedge\left(\left\langle b, b^{\prime}\right\rangle \leftarrow\left\langle c, c^{\prime}\right\rangle\right)\right] \vee\left[\left(\left\langle a, a^{\prime}\right\rangle \leftarrow\left\langle c, c^{\prime}\right\rangle\right) \wedge\right.$ $\left.\left(\left\langle b, b^{\prime}\right\rangle \leftrightarrow\left\langle c, c^{\prime}\right\rangle\right)\right]$

$=\left[\left(\left\langle b, b^{\prime}\right\rangle \leftarrow\left\langle c, c^{\prime}\right\rangle\right) \wedge\left(\left\langle a, a^{\prime}\right\rangle \leftrightarrow\left\langle c, c^{\prime}\right\rangle\right)\right] \vee\left[\left(\left\langle a, a^{\prime}\right\rangle \leftarrow\left\langle c, c^{\prime}\right\rangle\right) \wedge\right.$ $\left.\left(\left\langle b, b^{\prime}\right\rangle \leftrightarrow\left\langle c, c^{\prime}\right\rangle\right)\right]$.

It proves $(i)$.

By using Lemma 2.3 and Lemma 2.4

(ii). $\left(\left\langle a, a^{\prime}\right\rangle \vee\left\langle b, b^{\prime}\right\rangle\right) \leftrightarrow\left\langle c, c^{\prime}\right\rangle$

$=\left[\left(\left\langle a, a^{\prime}\right\rangle \vee\left\langle b, b^{\prime}\right\rangle\right) \leftarrow\left\langle c, c^{\prime}\right\rangle\right] \wedge\left[\left(\left\langle a, a^{\prime}\right\rangle \vee\left\langle b, b^{\prime}\right\rangle\right) \rightarrow\left\langle c, c^{\prime}\right\rangle\right]$

$=\left[\left(\left\langle a, a^{\prime}\right\rangle \vee\left\langle b, b^{\prime}\right\rangle\right) \rightarrow\left\langle c, c^{\prime}\right\rangle\right] \wedge\left[\left(\left\langle a, a^{\prime}\right\rangle \vee\left\langle b, b^{\prime}\right\rangle\right) \leftarrow\left\langle c, c^{\prime}\right\rangle\right]$

$=\left[\left(\left\langle a, a^{\prime}\right\rangle \rightarrow\left\langle c, c^{\prime}\right\rangle\right) \wedge\left(\left\langle b, b^{\prime}\right\rangle \rightarrow\left\langle c, c^{\prime}\right\rangle\right)\right] \wedge\left[\left(\left\langle a, a^{\prime}\right\rangle \leftarrow\left\langle c, c^{\prime}\right\rangle\right) \vee\right.$ $\left.\left(\left\langle b, b^{\prime}\right\rangle \leftarrow\left\langle c, c^{\prime}\right\rangle\right)\right]$

$=\left(\left\langle a, a^{\prime}\right\rangle \rightarrow\left\langle c, c^{\prime}\right\rangle\right) \wedge\left[\left[\left(\left\langle b, b^{\prime}\right\rangle \rightarrow\left\langle c, c^{\prime}\right\rangle\right) \wedge\left(\left\langle a, a^{\prime}\right\rangle \leftarrow\left\langle c, c^{\prime}\right\rangle\right)\right] \vee\right.$ $\left.\left(\left\langle b, b^{\prime}\right\rangle \rightarrow\left\langle c, c^{\prime}\right\rangle\right) \wedge\left(\left\langle b, b^{\prime}\right\rangle \leftarrow\left\langle c, c^{\prime}\right\rangle\right)\right]$

$=\left(\left\langle a, a^{\prime}\right\rangle \rightarrow\left\langle c, c^{\prime}\right\rangle\right) \wedge\left[\left[\left(\left\langle b, b^{\prime}\right\rangle \rightarrow\left\langle c, c^{\prime}\right\rangle\right) \wedge\left(\left\langle a, a^{\prime}\right\rangle \leftarrow\left\langle c, c^{\prime}\right\rangle\right)\right] \vee\right.$ $\left.\left[\left(\left\langle b, b^{\prime}\right\rangle \leftrightarrow\left\langle c, c^{\prime}\right\rangle\right)\right]\right]$ $=\left[\left(\left\langle a, a^{\prime}\right\rangle \rightarrow\left\langle c, c^{\prime}\right\rangle\right) \wedge\left(\left\langle b, b^{\prime}\right\rangle \rightarrow\left\langle c, c^{\prime}\right\rangle\right) \wedge\left(\left\langle a, a^{\prime}\right\rangle \leftarrow\left\langle c, c^{\prime}\right\rangle\right)\right] \vee$ $\left[\left(\left\langle a, a^{\prime}\right\rangle \rightarrow\left\langle c, c^{\prime}\right\rangle\right) \wedge\left(\left\langle b, b^{\prime}\right\rangle \leftrightarrow\left\langle c, c^{\prime}\right\rangle\right)\right]$

$=\left[\left(\left\langle a, a^{\prime}\right\rangle \leftrightarrow\left\langle c, c^{\prime}\right\rangle\right) \wedge\left(\left\langle b, b^{\prime}\right\rangle \rightarrow\left\langle c, c^{\prime}\right\rangle\right)\right] \vee\left[\left(\left\langle a, a^{\prime}\right\rangle \rightarrow\left\langle c, c^{\prime}\right\rangle\right) \wedge\right.$ $\left.\left(\left\langle b, b^{\prime}\right\rangle \leftrightarrow\left\langle c, c^{\prime}\right\rangle\right)\right]$

It proves $(i i)$.

Proposition 2.13. Let $\left\langle a, a^{\prime}\right\rangle,\left\langle b, b^{\prime}\right\rangle\left\langle c, c^{\prime}\right\rangle \in I F S$ then (i). $\left(\left\langle a, a^{\prime}\right\rangle \leftrightarrow\left\langle c, c^{\prime}\right\rangle\right) \wedge\left(\left\langle b, b^{\prime}\right\rangle \leftrightarrow\left\langle c, c^{\prime}\right\rangle\right) \leq\left(\left\langle a, a^{\prime}\right\rangle \wedge\left\langle b, b^{\prime}\right\rangle\right) \leftrightarrow$ $\left\langle c, c^{\prime}\right\rangle \leq\left(\left\langle a, a^{\prime}\right\rangle \leftrightarrow\left\langle c, c^{\prime}\right\rangle\right) \vee\left(\left\langle b, b^{\prime}\right\rangle \leftrightarrow\left\langle c, c^{\prime}\right\rangle\right)$

(ii). $\left(\left\langle a, a^{\prime}\right\rangle \leftrightarrow\left\langle c, c^{\prime}\right\rangle\right) \wedge\left(\left\langle b, b^{\prime}\right\rangle \leftrightarrow\left\langle c, c^{\prime}\right\rangle\right) \leq\left(\left\langle a, a^{\prime}\right\rangle \vee\right.$ $\left.\left\langle b, b^{\prime}\right\rangle\right) \leftrightarrow\left\langle c, c^{\prime}\right\rangle \leq\left(\left\langle a, a^{\prime}\right\rangle \leftrightarrow\left\langle c, c^{\prime}\right\rangle\right) \vee\left(\left\langle b, b^{\prime}\right\rangle \leftrightarrow\left\langle c, c^{\prime}\right\rangle\right)$

Proof. (i). $\left(\left\langle a, a^{\prime}\right\rangle \wedge\left\langle b, b^{\prime}\right\rangle\right) \leftrightarrow\left\langle c, c^{\prime}\right\rangle$

$\leq\left(\left\langle a, a^{\prime}\right\rangle \leftrightarrow\left\langle c, c^{\prime}\right\rangle\right) \vee\left(\left\langle b, b^{\prime}\right\rangle \leftrightarrow\left\langle c, c^{\prime}\right\rangle\right)$ is trivial from Proposition 2.3(i)

case 1. If $\left\langle a, a^{\prime}\right\rangle>\left\langle b, b^{\prime}\right\rangle$ then

$\left(\left\langle a, a^{\prime}\right\rangle \leftrightarrow\left\langle c, c^{\prime}\right\rangle\right) \wedge\left(\left\langle b, b^{\prime}\right\rangle \leftrightarrow\left\langle c, c^{\prime}\right\rangle\right)$

$= \begin{cases}\left(\left\langle a, a^{\prime}\right\rangle \leftrightarrow\left\langle c, c^{\prime}\right\rangle\right) \wedge\left\langle c, c^{\prime}\right\rangle & \text { if }\left\langle b, b^{\prime}\right\rangle>\left\langle c, c^{\prime}\right\rangle \\ \left\langle c, c^{\prime}\right\rangle & \text { if }\left\langle b, b^{\prime}\right\rangle=\left\langle c, c^{\prime}\right\rangle \\ \left(\left\langle a, a^{\prime}\right\rangle \leftrightarrow\left\langle c, c^{\prime}\right\rangle\right) \wedge\left\langle b, b^{\prime}\right\rangle & \text { if }\left\langle b, b^{\prime}\right\rangle<\left\langle c, c^{\prime}\right\rangle\end{cases}$

$\left(\left\langle a, a^{\prime}\right\rangle \wedge\left\langle b, b^{\prime}\right\rangle\right) \leftrightarrow\left\langle c, c^{\prime}\right\rangle=\left\langle b, b^{\prime}\right\rangle \leftrightarrow\left\langle c, c^{\prime}\right\rangle$

$= \begin{cases}\left\langle c, c^{\prime}\right\rangle & \text { if }\left\langle b, b^{\prime}\right\rangle>\left\langle c, c^{\prime}\right\rangle \\ \langle 1,0\rangle & \text { if }\left\langle b, b^{\prime}\right\rangle=\left\langle c, c^{\prime}\right\rangle \\ \left\langle b, b^{\prime}\right\rangle & \text { if }\left\langle b, b^{\prime}\right\rangle<\left\langle c, c^{\prime}\right\rangle\end{cases}$

From (2.5) and (2.6) $\left(\left\langle a, a^{\prime}\right\rangle \leftrightarrow\left\langle c, c^{\prime}\right\rangle\right) \wedge\left(\left\langle b, b^{\prime}\right\rangle \leftrightarrow\left\langle c, c^{\prime}\right\rangle\right) \leq$ $\left(\left\langle a, a^{\prime}\right\rangle \wedge\left\langle b, b^{\prime}\right\rangle\right) \leftrightarrow\left\langle c, c^{\prime}\right\rangle$.

case 2. If $\left\langle a, a^{\prime}\right\rangle=\left\langle b, b^{\prime}\right\rangle$, then

$\left(\left\langle a, a^{\prime}\right\rangle \leftrightarrow\left\langle c, c^{\prime}\right\rangle\right) \wedge\left(\left\langle b, b^{\prime}\right\rangle \quad \leftrightarrow \quad\left\langle c, c^{\prime}\right\rangle\right)=$

$\int\left\langle c, c^{\prime}\right\rangle$ if $\left.\left\langle b, b^{\prime}\right\rangle\right\rangle\left\langle c, c^{\prime}\right\rangle$

$\left\{\langle 1,0\rangle\right.$ if $\left\langle b, b^{\prime}\right\rangle=\left\langle c, c^{\prime}\right\rangle$

$\left\langle\left\langle, b^{\prime}\right\rangle\right.$ if $\left\langle b, b^{\prime}\right\rangle\left\langle\left\langle c, c^{\prime}\right\rangle\right.$

$=\left(\left\langle a, a^{\prime}\right\rangle \wedge\left\langle b, b^{\prime}\right\rangle\right) \leftrightarrow\left\langle c, c^{\prime}\right\rangle$.

case 3. If $\left\langle a, a^{\prime}\right\rangle\left\langle\left\langle b, b^{\prime}\right\rangle\right.$

$\left(\left\langle a, a^{\prime}\right\rangle \leftrightarrow\left\langle c, c^{\prime}\right\rangle\right) \wedge\left(\left\langle b, b^{\prime}\right\rangle \quad \leftrightarrow \quad\left\langle c, c^{\prime}\right\rangle\right)=$

$\left.\int\left\langle c, c^{\prime}\right\rangle \wedge\left(\left\langle b, b^{\prime}\right\rangle \leftrightarrow\left\langle c, c^{\prime}\right\rangle\right)\right)$ if $\left.\left\langle a, a^{\prime}\right\rangle\right\rangle\left\langle c, c^{\prime}\right\rangle$

$\left\{\left\langle c, c^{\prime}\right\rangle\right.$ if $\left\langle a, a^{\prime}\right\rangle=\left\langle c, c^{\prime}\right\rangle$

$\left\langle\left\langle a, a^{\prime}\right\rangle \wedge\left(\left\langle b, b^{\prime}\right\rangle \leftrightarrow\left\langle c, c^{\prime}\right\rangle\right)\right.$ if $\left\langle a, a^{\prime}\right\rangle\left\langle\left\langle c, c^{\prime}\right\rangle\right.$

$\left(\left\langle a, a^{\prime}\right\rangle \wedge\left\langle b, b^{\prime}\right\rangle\right) \leftrightarrow\left\langle c, c^{\prime}\right\rangle= \begin{cases}\left\langle c, c^{\prime}\right\rangle & \text { if }\left\langle a, a^{\prime}\right\rangle>\left\langle c, c^{\prime}\right\rangle \\ \langle 1,0\rangle & \text { if }\left\langle a, a^{\prime}\right\rangle=\left\langle c, c^{\prime}\right\rangle \\ \left\langle a, a^{\prime}\right\rangle & \text { if }\left\langle a, a^{\prime}\right\rangle<\left\langle c, c^{\prime}\right\rangle\end{cases}$

By (2.7) and (2.8) $\left(\left\langle a, a^{\prime}\right\rangle \leftrightarrow\left\langle c, c^{\prime}\right\rangle\right) \wedge\left(\left\langle b, b^{\prime}\right\rangle \leftrightarrow\left\langle c, c^{\prime}\right\rangle\right) \leq$ $\left(\left\langle a, a^{\prime}\right\rangle \wedge\left\langle b, b^{\prime}\right\rangle\right) \leftrightarrow\left\langle c, c^{\prime}\right\rangle$.

Through compared with we can see for any $\left\langle a, a^{\prime}\right\rangle,\left\langle b, b^{\prime}\right\rangle,\left\langle c, c^{\prime}\right\rangle \in$ IFS

$\left(\left\langle a, a^{\prime}\right\rangle \leftrightarrow\left\langle c, c^{\prime}\right\rangle\right) \wedge\left(\left\langle b, b^{\prime}\right\rangle \leftrightarrow\left\langle c, c^{\prime}\right\rangle\right) \leq\left(\left\langle a, a^{\prime}\right\rangle \wedge\left\langle b, b^{\prime}\right\rangle\right) \leftrightarrow$ $\left(\left\langle c, c^{\prime}\right\rangle \leq\left(\left\langle a, a^{\prime}\right\rangle \leftrightarrow\left\langle c, c^{\prime}\right\rangle\right) \vee\left(\left\langle b, b^{\prime}\right\rangle \leftrightarrow\left\langle c, c^{\prime}\right\rangle\right)\right.$

(ii). By Proposition 2.3(ii) it is trivial that $\left(\left\langle a, a^{\prime}\right\rangle \vee\left\langle b, b^{\prime}\right\rangle\right) \leftrightarrow$ $\left\langle c, c^{\prime}\right\rangle \$ \leq\left(\left\langle a, a^{\prime}\right\rangle \leftrightarrow\left\langle c, c^{\prime}\right\rangle\right) \vee\left(\left\langle b, b^{\prime}\right\rangle \leftrightarrow\left\langle c, c^{\prime}\right\rangle\right)$

If $\left.\left\langle a, a^{\prime}\right\rangle\right\rangle\left\langle b, b^{\prime}\right\rangle$ then

$\left(\left\langle a, a^{\prime}\right\rangle \leftrightarrow\left\langle c, c^{\prime}\right\rangle\right) \wedge\left(\left\langle b, b^{\prime}\right\rangle \quad \leftrightarrow \quad\left\langle c, c^{\prime}\right\rangle\right)=$ $\left\{\begin{array}{l}\left(\left\langle a, a^{\prime}\right\rangle \leftrightarrow\left\langle c, c^{\prime}\right\rangle\right) \wedge\left\langle c, c^{\prime}\right\rangle \text { if }\left\langle b, b^{\prime}\right\rangle>\left\langle c, c^{\prime}\right\rangle \\ \left\langle c, c^{\prime}\right\rangle \text { if }\left\langle b, b^{\prime}\right\rangle=\left\langle c, c^{\prime}\right\rangle \\ \left(\left\langle a, a^{\prime}\right\rangle \leftrightarrow\left\langle c, c^{\prime}\right\rangle\right) \wedge\left\langle b, b^{\prime}\right\rangle \text { if }\left\langle b, b^{\prime}\right\rangle\left\langle\left\langle c, c^{\prime}\right\rangle\right.\end{array}\right.$ 
$\left(\left\langle a, a^{\prime}\right\rangle \vee\left\langle b, b^{\prime}\right\rangle\right) \leftrightarrow\left\langle c, c^{\prime}\right\rangle=\left(\left\langle a, a^{\prime}\right\rangle \leftrightarrow\left\langle c, c^{\prime}\right\rangle\right)=$
$\left\{\begin{array}{lll}\left\langle c, c^{\prime}\right\rangle & \text { if }\left\langle b, b^{\prime}\right\rangle>\left\langle c, c^{\prime}\right\rangle \\ \left\langle c, c^{\prime}\right\rangle & \text { if }\left\langle b, b^{\prime}\right\rangle=\left\langle c, c^{\prime}\right\rangle & \\ \left\langle a, a^{\prime}\right\rangle \leftrightarrow\left\langle c, c^{\prime}\right\rangle & \text { if }\left\langle b, b^{\prime}\right\rangle\left\langle\left\langle c, c^{\prime}\right\rangle\right. & \end{array}\right.$

By (2.9) and (2.10) $\left(\left\langle a, a^{\prime}\right\rangle \leftrightarrow\left\langle c, c^{\prime}\right\rangle\right) \wedge\left(\left\langle b, b^{\prime}\right\rangle \leftrightarrow\left\langle c, c^{\prime}\right\rangle\right) \leq$ $\left(\left\langle a, a^{\prime}\right\rangle \vee\left\langle b, b^{\prime}\right\rangle\right) \leftrightarrow\left\langle c, c^{\prime}\right\rangle$

case 2. If $\left\langle a, a^{\prime}\right\rangle=\left\langle b, b^{\prime}\right\rangle$

$\left(\left\langle a, a^{\prime}\right\rangle \leftrightarrow\left\langle c, c^{\prime}\right\rangle\right) \wedge\left(\left\langle b, b^{\prime}\right\rangle \leftrightarrow\left\langle c, c^{\prime}\right\rangle\right)$

$=\left\{\begin{array}{l}\left(\left\langle a, a^{\prime}\right\rangle \leftrightarrow\left\langle c, c^{\prime}\right\rangle\right) \wedge\left\langle c, c^{\prime}\right\rangle=\left\langle c, c^{\prime}\right\rangle \\ \left.\text { if }\left\langle b, b^{\prime}\right\rangle\right\rangle\left\langle c, c^{\prime}\right\rangle \\ \left(\left\langle a, a^{\prime}\right\rangle \leftrightarrow\left\langle c, c^{\prime}\right\rangle\right)=\langle 1,0\rangle \\ \text { if }\left\langle b, b^{\prime}\right\rangle=\left\langle c, c^{\prime}\right\rangle \\ \left(\left\langle a, a^{\prime}\right\rangle \leftrightarrow\left\langle c, c^{\prime}\right\rangle\right) \wedge\left\langle b, b^{\prime}\right\rangle=\left\langle b, b^{\prime}\right\rangle \\ \text { if }\left\langle b, b^{\prime}\right\rangle<\left\langle c, c^{\prime}\right\rangle\end{array}\right.$

$\left(\left\langle a, a^{\prime}\right\rangle \vee\left\langle b, b^{\prime}\right\rangle\right) \leftrightarrow\left\langle c, c^{\prime}\right\rangle=\left(\left\langle b, b^{\prime}\right\rangle \leftrightarrow\left\langle c, c^{\prime}\right\rangle\right)=$

$\left\langle\left\langle c, c^{\prime}\right\rangle\right.$ if $\left.\left\langle b, b^{\prime}\right\rangle\right\rangle\left\langle c, c^{\prime}\right\rangle$

$\left\{\langle 1,0\rangle\right.$ if $\left\langle b, b^{\prime}\right\rangle=\left\langle c, c^{\prime}\right\rangle$

$\left\langle\left\langle b, b^{\prime}\right\rangle\right.$ if $\left\langle b, b^{\prime}\right\rangle<\left\langle c, c^{\prime}\right\rangle$

Thus $\left(\left\langle a, a^{\prime}\right\rangle \leftrightarrow\left\langle c, c^{\prime}\right\rangle\right) \wedge\left(\left\langle b, b^{\prime}\right\rangle \leftrightarrow\left\langle c, c^{\prime}\right\rangle\right) \leq$ $\left(\left\langle a, a^{\prime}\right\rangle \vee\left\langle b, b^{\prime}\right\rangle\right) \leftrightarrow\left\langle c, c^{\prime}\right\rangle$

case 3. If $\left\langle a, a^{\prime}\right\rangle\left\langle\left\langle b, b^{\prime}\right\rangle\right.$ then

$\left(\left\langle a, a^{\prime}\right\rangle \leftrightarrow\left\langle c, c^{\prime}\right\rangle\right) \wedge\left(\left\langle b, b^{\prime}\right\rangle \quad \leftrightarrow \quad\left\langle c, c^{\prime}\right\rangle\right)=$

$\int\left\langle c, c^{\prime}\right\rangle \wedge\left(\left\langle b, b^{\prime}\right\rangle \leftrightarrow\left\langle c, c^{\prime}\right\rangle\right)$ if $\left.\left\langle a, a^{\prime}\right\rangle\right\rangle\left\langle c, c^{\prime}\right\rangle$

$\begin{cases}\left\langle c, c^{\prime}\right\rangle & \text { if }\left\langle a, a^{\prime}\right\rangle=\left\langle c, c^{\prime}\right\rangle\end{cases}$

$\left\langle\left\langle a, a^{\prime}\right\rangle \wedge\left(\left\langle b, b^{\prime}\right\rangle \leftrightarrow\left\langle c, c^{\prime}\right\rangle\right)\right.$ if $\left\langle a, a^{\prime}\right\rangle\left\langle\left\langle c, c^{\prime}\right\rangle\right.$

$\left(\left\langle a, a^{\prime}\right\rangle \vee\left\langle b, b^{\prime}\right\rangle\right) \leftrightarrow\left\langle c, c^{\prime}\right\rangle=\left(\left\langle b, b^{\prime}\right\rangle \leftrightarrow\left\langle c, c^{\prime}\right\rangle\right)=$ $\begin{cases}\left\langle c, c^{\prime}\right\rangle & \left.\text { if }\left\langle a, a^{\prime}\right\rangle\right\rangle\left\langle c, c^{\prime}\right\rangle \\ \left\langle c, c^{\prime}\right\rangle & \text { if }\left\langle a, a^{\prime}\right\rangle=\left\langle c, c^{\prime}\right\rangle \\ \left\langle b, b^{\prime}\right\rangle \leftrightarrow\left\langle c, c^{\prime}\right\rangle & \text { if }\left\langle a, a^{\prime}\right\rangle<\left\langle c, c^{\prime}\right\rangle\end{cases}$

By (2.11) and (2.12) $\left(\left\langle a, a^{\prime}\right\rangle \leftrightarrow\left\langle c, c^{\prime}\right\rangle\right) \wedge\left(\left\langle b, b^{\prime}\right\rangle \leftrightarrow\left\langle c, c^{\prime}\right\rangle\right) \leq$ $\left(\left\langle a, a^{\prime}\right\rangle \vee\left\langle b, b^{\prime}\right\rangle\right) \leftrightarrow\left\langle c, c^{\prime}\right\rangle$.

Proposition 2.14. Let $\left\langle a, a^{\prime}\right\rangle,\left\langle b, b^{\prime}\right\rangle$ and $\left\langle c, c^{\prime}\right\rangle \in$ IFS if $\left\langle a, a^{\prime}\right\rangle \wedge\left\langle b, b^{\prime}\right\rangle \neq\left\langle c, c^{\prime}\right\rangle$ then

$(i) .\left(\left\langle a, a^{\prime}\right\rangle \wedge\left\langle b, b^{\prime}\right\rangle\right) \leftrightarrow\left\langle c, c^{\prime}\right\rangle=\left(\left\langle a, a^{\prime}\right\rangle \leftrightarrow\left\langle c, c^{\prime}\right\rangle\right) \wedge\left(\left\langle b, b^{\prime}\right\rangle \leftrightarrow\right.$ $\left.\left\langle c, c^{\prime}\right\rangle\right)$

$(i i) .\left(\left\langle a, a^{\prime}\right\rangle \vee\left\langle b, b^{\prime}\right\rangle\right) \leftrightarrow\left\langle c, c^{\prime}\right\rangle=\left(\left\langle a, a^{\prime}\right\rangle \leftrightarrow\left\langle c, c^{\prime}\right\rangle\right) \vee\left(\left\langle b, b^{\prime}\right\rangle \leftrightarrow\right.$ $\left.\left\langle c, c^{\prime}\right\rangle\right)$

Proof. (i). If $\left.\left(\left\langle a, a^{\prime}\right\rangle \wedge\left\langle b, b^{\prime}\right\rangle\right)\right\rangle\left\langle c, c^{\prime}\right\rangle$

then $\left(\left\langle a, a^{\prime}\right\rangle \wedge\left\langle b, b^{\prime}\right\rangle\right) \leftrightarrow\left\langle c, c^{\prime}\right\rangle=\left\langle c, c^{\prime}\right\rangle$

while $\left(\left\langle a, a^{\prime}\right\rangle \leftrightarrow\left\langle c, c^{\prime}\right\rangle\right) \wedge\left(\left\langle b, b^{\prime}\right\rangle \leftrightarrow\left\langle c, c^{\prime}\right\rangle\right)=\left\langle c, c^{\prime}\right\rangle \wedge\left\langle c, c^{\prime}\right\rangle=$ $\left\langle c, c^{\prime}\right\rangle$

if $\left(\left\langle a, a^{\prime}\right\rangle \wedge\left\langle b, b^{\prime}\right\rangle\right)<\left\langle c, c^{\prime}\right\rangle$ then $\left\langle a, a^{\prime}\right\rangle\left\langle\left\langle c, c^{\prime}\right\rangle\right.$ or $\left\langle b, b^{\prime}\right\rangle\left\langle\left\langle c, c^{\prime}\right\rangle\right.$ suppose $\left\langle a, a^{\prime}\right\rangle<\left\langle c, c^{\prime}\right\rangle$

then $\left(\left\langle a, a^{\prime}\right\rangle \wedge\left\langle b, b^{\prime}\right\rangle\right) \leftrightarrow\left\langle c, c^{\prime}\right\rangle=\left\langle a, a^{\prime}\right\rangle \wedge\left\langle b, b^{\prime}\right\rangle=$

$\int\left\langle b, b^{\prime}\right\rangle \quad$ if $\left\langle a, a^{\prime}\right\rangle \geq\left\langle b, b^{\prime}\right\rangle$

$\left\{\left\langle a, a^{\prime}\right\rangle \quad\right.$ if $\left\langle a, a^{\prime}\right\rangle<\left\langle b, b^{\prime}\right\rangle$

$\left(\left\langle a, a^{\prime}\right\rangle \leftrightarrow\left\langle c, c^{\prime}\right\rangle\right) \wedge\left(\left\langle b, b^{\prime}\right\rangle \leftrightarrow\left\langle c, c^{\prime}\right\rangle\right)$

$=\left\langle a, a^{\prime}\right\rangle \wedge\left(\left\langle b, b^{\prime}\right\rangle \leftrightarrow\left\langle c, c^{\prime}\right\rangle\right)$

$= \begin{cases}\left\langle b, b^{\prime}\right\rangle & \text { if }\left\langle a, a^{\prime}\right\rangle \geq\left\langle b, b^{\prime}\right\rangle \\ \left\langle a, a^{\prime}\right\rangle & \text { if }\left\langle a, a^{\prime}\right\rangle<\left\langle b, b^{\prime}\right\rangle\end{cases}$

hence $\left(\left\langle a, a^{\prime}\right\rangle \wedge\left\langle b, b^{\prime}\right\rangle\right) \leftrightarrow\left\langle c, c^{\prime}\right\rangle=\left(\left\langle a, a^{\prime}\right\rangle \leftrightarrow\left\langle c, c^{\prime}\right\rangle\right) \wedge\left(\left\langle b, b^{\prime}\right\rangle \leftrightarrow\right.$ $\left.\left\langle c, c^{\prime}\right\rangle\right)$.

(ii). Since $\left(\left\langle a, a^{\prime}\right\rangle \wedge\left\langle b, b^{\prime}\right\rangle\right) \neq\left\langle c, c^{\prime}\right\rangle$ then suppose $\left(\left\langle a, a^{\prime}\right\rangle \neq\right.$ $\left.\left\langle c, c^{\prime}\right\rangle\right)$ and $\left(\left\langle b, b^{\prime}\right\rangle=\left\langle c, c^{\prime}\right\rangle\right) \Rightarrow\left\langle a, a^{\prime}\right\rangle\left\langle\left\langle c, c^{\prime}\right\rangle=\left\langle b, b^{\prime}\right\rangle\right.$ in

\section{this case}

$\left(\left\langle a, a^{\prime}\right\rangle \vee\left\langle b, b^{\prime}\right\rangle\right) \leftrightarrow\left\langle c, c^{\prime}\right\rangle=\left(\left\langle b, b^{\prime}\right\rangle \leftrightarrow\left\langle c, c^{\prime}\right\rangle\right)=\left(\left\langle c, c^{\prime}\right\rangle \leftrightarrow\right.$ $\left.\left\langle c, c^{\prime}\right\rangle\right)=\langle 1,0\rangle$

$\left(\left\langle a, a^{\prime}\right\rangle \leftrightarrow\left\langle c, c^{\prime}\right\rangle\right) \vee\left(\left\langle b, b^{\prime}\right\rangle \leftrightarrow\left\langle c, c^{\prime}\right\rangle\right)=\left(\left\langle a, a^{\prime}\right\rangle \leftrightarrow\left\langle c, c^{\prime}\right\rangle\right) \vee\langle 1,0\rangle$ $=\left\langle a, a^{\prime}\right\rangle \vee\langle 1,0\rangle=\langle 1,0\rangle$

hence $(i i)$ holds.

If $\left\langle a, a^{\prime}\right\rangle=\left\langle b, b^{\prime}\right\rangle$ then

$\left(\left\langle a, a^{\prime}\right\rangle \vee\left\langle b, b^{\prime}\right\rangle\right) \leftrightarrow\left\langle c, c^{\prime}\right\rangle=\left(\left\langle b, b^{\prime}\right\rangle \vee\left\langle b, b^{\prime}\right\rangle\right) \leftrightarrow\left\langle c, c^{\prime}\right\rangle=$ $\left\langle b, b^{\prime}\right\rangle \leftrightarrow\left\langle c, c^{\prime}\right\rangle$

$\left(\left\langle a, a^{\prime}\right\rangle \leftrightarrow\left\langle c, c^{\prime}\right\rangle\right) \vee\left(\left\langle b, b^{\prime}\right\rangle \leftrightarrow\left\langle c, c^{\prime}\right\rangle\right)$

$=\left(\left\langle b, b^{\prime}\right\rangle \leftrightarrow\left\langle c, c^{\prime}\right\rangle\right) \vee\left(\left\langle b, b^{\prime}\right\rangle \leftrightarrow\left\langle c, c^{\prime}\right\rangle\right)$

$=\left(\left\langle b, b^{\prime}\right\rangle \leftrightarrow\left\langle c, c^{\prime}\right\rangle\right)$

$\left.\left(\left\langle a, a^{\prime}\right\rangle\right) \vee\left\langle b, b^{\prime}\right\rangle\right) \leftrightarrow\left\langle c, c^{\prime}\right\rangle=\left(\left\langle a, a^{\prime}\right\rangle \leftrightarrow\left\langle c, c^{\prime}\right\rangle\right) \vee\left(\left\langle b, b^{\prime}\right\rangle \leftrightarrow\right.$ $\left.\left\langle c, c^{\prime}\right\rangle\right)$

Hence ( $i i$ ) holds. Similarly we can prove the other cases also.

Proposition 2.15. Let $\left\langle a, a^{\prime}\right\rangle,\left\langle b, b^{\prime}\right\rangle$ and $\left\langle c, c^{\prime}\right\rangle \in I F S$ and $\left\langle c, c^{\prime}\right\rangle \neq\langle 1,0\rangle$

then $\left(\left\langle a, a^{\prime}\right\rangle \wedge\left\langle b, b^{\prime}\right\rangle\right) \leftrightarrow\left\langle c, c^{\prime}\right\rangle=\left(\left\langle a, a^{\prime}\right\rangle \leftrightarrow\left\langle c, c^{\prime}\right\rangle\right) \wedge\left(\left\langle b, b^{\prime}\right\rangle \leftrightarrow\right.$ $\left.\left\langle c, c^{\prime}\right\rangle\right)$

if and only if one of the following condition holds

(i). When $\left\langle a, a^{\prime}\right\rangle>\left\langle b, b^{\prime}\right\rangle,\left\langle b, b^{\prime}\right\rangle \neq\left\langle c, c^{\prime}\right\rangle$

(ii). When $\left\langle a, a^{\prime}\right\rangle<\left\langle b, b^{\prime}\right\rangle,\left\langle a, a^{\prime}\right\rangle \neq\left\langle c, c^{\prime}\right\rangle$

(iii). When $\left\langle a, a^{\prime}\right\rangle=\left\langle b, b^{\prime}\right\rangle$

Proof. (i) $\left.\left\langle a, a^{\prime}\right\rangle\right\rangle\left\langle b, b^{\prime}\right\rangle$

If $\left\langle b, b^{\prime}\right\rangle=\left\langle c, c^{\prime}\right\rangle$

$\left(\left\langle a, a^{\prime}\right\rangle \wedge\left\langle b, b^{\prime}\right\rangle\right) \leftrightarrow\left\langle c, c^{\prime}\right\rangle=\left\langle b, b^{\prime}\right\rangle \leftrightarrow\left\langle c, c^{\prime}\right\rangle=\langle 1,0\rangle$

$\left.\left(\left\langle a, a^{\prime}\right\rangle\right) \leftrightarrow\left\langle c, c^{\prime}\right\rangle\right) \wedge\left(\left\langle b, b^{\prime}\right\rangle \leftrightarrow\left\langle c, c^{\prime}\right\rangle\right)$

$\left.=\left(\left\langle a, a^{\prime}\right\rangle\right) \leftrightarrow\left\langle c, c^{\prime}\right\rangle\right) \wedge\left(\left\langle c, c^{\prime}\right\rangle \leftrightarrow\left\langle c, c^{\prime}\right\rangle\right)$

$\left.\left.=\left(\left\langle a, a^{\prime}\right\rangle \leftrightarrow\left\langle b, b^{\prime}\right\rangle\right) \wedge\langle 1,0\rangle=\left\langle b, b^{\prime}\right\rangle\right) \wedge\langle 1,0\rangle=\left(\left\langle c, c^{\prime}\right\rangle\right) \wedge\langle 1,0\rangle\right)=$ $\left\langle c, c^{\prime}\right\rangle$

but $\left\langle c, c^{\prime}\right\rangle \neq\langle 1,0\rangle$ a contradiction

(ii). Assume $\left\langle a, a^{\prime}\right\rangle<\left\langle b, b^{\prime}\right\rangle$

if $\left\langle a, a^{\prime}\right)=\left\langle c, c^{\prime}\right\rangle$

$\left(\left\langle a, a^{\prime}\right\rangle \wedge\left\langle b, b^{\prime}\right\rangle\right) \leftrightarrow\left\langle c, c^{\prime}\right\rangle=\left\langle a, a^{\prime}\right\rangle \leftrightarrow\left\langle c, c^{\prime}\right\rangle$

$=\left\langle c, c^{\prime}\right\rangle \leftrightarrow\left\langle c, c^{\prime}\right\rangle=\langle 1,0\rangle$

$\left(\left\langle a, a^{\prime}\right\rangle \leftrightarrow\left\langle c, c^{\prime}\right\rangle\right) \wedge\left(\left\langle b, b^{\prime}\right\rangle \leftrightarrow\left\langle c, c^{\prime}\right\rangle\right)=\left(\left\langle c, c^{\prime}\right\rangle \leftrightarrow\left\langle c, c^{\prime}\right\rangle\right) \wedge$ $\left(\left\langle b, b^{\prime}\right\rangle \leftrightarrow\left\langle a, a^{\prime}\right\rangle\right)=\langle 1,0\rangle \wedge\left(\left\langle b, b^{\prime}\right\rangle \leftrightarrow\left\langle a, a^{\prime}\right\rangle\right)=\langle 1,0\rangle \wedge\left\langle a, a^{\prime}\right\rangle$ $=\langle 1,0\rangle \wedge\left\langle c, c^{\prime}\right\rangle=\left\langle c, c^{\prime}\right\rangle$ but $\left\langle c, c^{\prime}\right\rangle \neq\langle 1,0\rangle$

which is a contradiction

(iii) When $\left\langle a, a^{\prime}\right\rangle=\left\langle b, b^{\prime}\right\rangle$

$\left(\left\langle a, a^{\prime}\right\rangle \wedge\left\langle b, b^{\prime}\right\rangle\right) \leftrightarrow\left\langle c, c^{\prime}\right\rangle=\left(\left\langle b, b^{\prime}\right\rangle \leftrightarrow\left\langle c, c^{\prime}\right\rangle\right)$

$=\left(\left\langle a, a^{\prime}\right\rangle \leftrightarrow\left\langle c, c^{\prime}\right\rangle\right) \wedge\left(\left\langle b, b^{\prime}\right\rangle \leftrightarrow\left\langle c, c^{\prime}\right\rangle\right)=\left(\left\langle b, b^{\prime}\right\rangle \leftrightarrow\left\langle c, c^{\prime}\right\rangle\right) \wedge$ $\left(\left\langle b, b^{\prime}\right\rangle \leftrightarrow\left\langle c, c^{\prime}\right\rangle\right)=\left(\left\langle b, b^{\prime}\right\rangle \leftrightarrow\left\langle c, c^{\prime}\right\rangle\right)$

therefore the conclusion is obvious.

PROPOSITION 2.16. Let $\left\langle a, a^{\prime}\right\rangle,\left\langle b, b^{\prime}\right\rangle$ and $\left\langle c, c^{\prime}\right\rangle \in I F S$ and $\left\langle c, c^{\prime}\right\rangle \neq\langle 1,0\rangle$

then $\left(\left\langle a, a^{\prime}\right\rangle \vee\left\langle b, b^{\prime}\right\rangle\right) \leftrightarrow\left\langle c, c^{\prime}\right\rangle=\left(\left\langle a, a^{\prime}\right\rangle \leftrightarrow\left\langle c, c^{\prime}\right\rangle\right) \vee\left(\left\langle b, b^{\prime}\right\rangle \leftrightarrow\right.$ $\left.\left\langle c, c^{\prime}\right\rangle\right)$

$\Leftrightarrow$ one of the following condition holds

(i). When $\left\langle a, a^{\prime}\right\rangle>\left\langle b, b^{\prime}\right\rangle,\left\langle b, b^{\prime}\right\rangle \neq\left\langle c, c^{\prime}\right\rangle$

(ii). When $\left\langle a, a^{\prime}\right\rangle\left\langle\left\langle b, b^{\prime}\right\rangle,\left\langle a, a^{\prime}\right\rangle \neq\left\langle c, c^{\prime}\right\rangle\right.$

(iii). When $\left\langle a, a^{\prime}\right\rangle=\left\langle b, b^{\prime}\right\rangle$

Proof. (i). If $\left.\left\langle a, a^{\prime}\right\rangle\right\rangle\left\langle b, b^{\prime}\right\rangle$

certainly $\left\langle b, b^{\prime}\right\rangle \neq\left\langle c, c^{\prime}\right\rangle$. Otherwise

$\left(\left\langle a, a^{\prime}\right\rangle \vee\left\langle b, b^{\prime}\right\rangle\right) \leftrightarrow\left\langle c, c^{\prime}\right\rangle=\left\langle a, a^{\prime}\right\rangle \leftrightarrow\left\langle c, c^{\prime}\right\rangle$

$=\left\langle a, a^{\prime}\right\rangle \leftrightarrow\left\langle b, b^{\prime}\right\rangle$

$=\left\langle b, b^{\prime}\right\rangle=\left\langle c, c^{\prime}\right\rangle$

$\left.\left.\left(\left\langle a, a^{\prime}\right\rangle\right) \leftrightarrow\left\langle c, c^{\prime}\right\rangle\right) \vee\left(\left\langle b, b^{\prime}\right\rangle\right) \leftrightarrow\left\langle c, c^{\prime}\right\rangle\right)$

$=\left(\left\langle a, a^{\prime}\right\rangle \leftrightarrow\left\langle b, b^{\prime}\right\rangle\right) \vee\langle 1,0\rangle=\left\langle b, b^{\prime}\right\rangle \vee\langle 1,0\rangle=\langle 1,0\rangle$ but $\left\langle c, c^{\prime}\right\rangle \neq$ $\langle 1,0\rangle$

a contradiction. 
(ii). When $\left\langle a, a^{\prime}\right\rangle\left\langle\left\langle b, b^{\prime}\right\rangle\right.$ we have $\left\langle a, a^{\prime}\right\rangle \neq\left\langle c, c^{\prime}\right\rangle$

otherwise $\left(\left\langle a, a^{\prime}\right\rangle \vee\left\langle b, b^{\prime}\right\rangle\right) \leftrightarrow\left\langle c, c^{\prime}\right\rangle=\left\langle b, b^{\prime}\right\rangle \leftrightarrow\left\langle c, c^{\prime}\right\rangle$

$=\left\langle b, b^{\prime}\right\rangle \leftrightarrow\left\langle a, a^{\prime}\right\rangle$

$=\left\langle a, a^{\prime}\right\rangle$

$\left(\left\langle a, a^{\prime}\right\rangle \leftrightarrow\left\langle c, c^{\prime}\right\rangle\right) \vee\left(\left\langle b, b^{\prime}\right\rangle \leftrightarrow\left\langle c, c^{\prime}\right\rangle\right)=\left(\left\langle c, c^{\prime}\right\rangle \leftrightarrow\left\langle c, c^{\prime}\right\rangle\right) \vee$

$\left(\left\langle b, b^{\prime}\right\rangle \leftrightarrow\left\langle c, c^{\prime}\right\rangle\right)$

$=\langle 1,0\rangle \vee\left(\left\langle b, b^{\prime}\right\rangle \leftrightarrow\left\langle a, a^{\prime}\right\rangle\right)$

$\langle 1,0\rangle \vee\left\langle a, a^{\prime}\right\rangle=\langle 1,0\rangle$

which is a contradiction.

(iii).If $\left\langle a, a^{\prime}\right\rangle=\left\langle b, b^{\prime}\right\rangle$ then

$\left(\left\langle a, a^{\prime}\right\rangle \vee\left\langle b, b^{\prime}\right\rangle\right) \leftrightarrow\left\langle c, c^{\prime}\right\rangle=\left\langle b, b^{\prime}\right\rangle \leftrightarrow\left\langle c, c^{\prime}\right\rangle$

$\left(\left\langle a, a^{\prime}\right\rangle \leftrightarrow\left\langle c, c^{\prime}\right\rangle\right) \vee\left(\left\langle b, b^{\prime}\right\rangle \leftrightarrow\left\langle c, c^{\prime}\right\rangle\right)$

$\left(\left\langle b, b^{\prime}\right\rangle \leftrightarrow\left\langle c, c^{\prime}\right\rangle\right) \vee\left(\left\langle b, b^{\prime}\right\rangle \leftrightarrow\left\langle c, c^{\prime}\right\rangle\right)=\left(\left\langle b, b^{\prime}\right\rangle \leftrightarrow\left\langle c, c^{\prime}\right\rangle\right)$.

Thus $($ iii $)$ holds.

\section{REFERENCES}

[1] Atanassov K., Intuitionistic Fuzzy Sets., VII ITKR's Section, Sofia, June 1983.

[2] Hashimoto H., Sub-inverses of fuzzy Matrices, Fuzzy Sets and Systems, Vol.12(1984),155-168.

[3] Murugadas P and Lalitha K., Dual Implication Operator in Intuitionistic Fuzzy Matrices, International conference on Mathematical Modelling and its Application-2012.

[4] Sriram S and Murugadas P., Sub-inverses of Intuitionistic Fuzzy Matrices, Acta Ciencia Indica(mathematics), Vol. XXXVII M,No.1,41(2011).

[5] Sriram S and P. Murugadas., On Semiring of Intuitionistic Fuzzy Matrices, Applied Mathematical Science, Vol. 4(23), (2010), 1099-1105.

[6] Sriram S and P. Murugadas., Contributions to a Study on Generalized Fuzzy Matrices, Ph.D Thesis, Department of Mathematics, Annamalai University, Tamil Nadu, India, (2011).

[7] Zadeh L.A., Fuzzy Sets, Journal of Information and Control, Vol.8(1965), 338-353. 